\title{
Minimally Invasive Surgery for Retroperitoneal Sarcoma: Just Because We Can Does Not Mean We Should
}

\author{
Alessandro Gronchi, $\mathrm{MD}^{1}$ (D), Aimee Crago, $\mathrm{MD}^{2}$, and Chandrajit P. Raut, MD, $\mathrm{MSc}^{3}$ \\ ${ }^{1}$ Department of Surgery, Fondazione IRCCS Istituto Nazionale dei Tumori, Milan, Italy; ${ }^{2}$ Gastric and Mixed Tumor \\ Service, Department of Surgery, Memorial Sloan-Kettering Cancer Center, New York, NY; ${ }^{3}$ Department of Surgery, \\ Center for Sarcoma and Bone Oncology, Dana-Farber Cancer Institute, Harvard Medical School, Brigham and Women's \\ Hospital, Boston, MA
}

We read with interest the recent article by Gani and coauthors ${ }^{1}$ about the use of minimally invasive surgery (MIS) for the management of primary retroperitoneal sarcoma (RPS). The authors put together an elegant analysis, utilizing the National Cancer Data Base (NCDB) to attempt to answer a question that cannot be readily addressed using prospective, single-institution databases. The limitations of this particular database are well highlighted in their manuscript, and they made several statistical efforts to minimize them. However, beyond the inherent limitations of the database itself, several issues, directly or indirectly analyzed and discussed, raise major concerns. A clear message needs to be delivered, and serves as the rationale for this editorial.

First, the percentage of RPS patients who are still primarily treated in the community and outside of highvolume centers in the US, as well as in several other countries, is worrisome; specialty consultation should be obtained whenever possible. Whatever approach (open or MIS), a surgeon having treated few of these patients may inadvertently undertreat his/her patients. Consultation of the available guidelines alone cannot compensate for the lack of experience and multidisciplinary evaluation. ${ }^{2}$ In fact, recent data have demonstrated that sarcoma patients undergoing evaluation in a specialized multidisciplinary tumor board had better local relapse-free and relapse-free survival compared with patients who did not. ${ }^{3}$ There is a

\section{(C) Society of Surgical Oncology 2018}

First Received: 2 June 2018;

Published Online: 14 June 2018

A. Gronchi, MD

e-mail: alessandro.gronchi@istitutotumori.mi.it growing body of evidence regarding the better outcome, specifically among RPS patients initially treated in specialized centers. ${ }^{4-7}$ This disease, or in reality, family of diseases, is complex. There are several histological subtypes, which have different behavior and risks. The anatomical location is also variable and challenging. The same approach is not universally applicable to all presentations and must be tailored to histologic subtype and radiographic subtleties. Furthermore, even experienced academic centers may not necessarily have surgeons specialized enough in the management of this disease. Indeed, this may have contributed to the reasons why no significant difference between overall survival after open surgery versus MIS was observed. Open surgery was indeed a proxy of surgery performed at an academic center, but whether the use of MIS was more common in low- or highvolume centers was not specified or investigated. Routine referral of these patients at initial presentation to sarcoma centers experienced in RPS care should be encouraged. The initial approach is critically important for maximizing the chance of cure. ${ }^{8}$ If the first operation is inadequate or suboptimal, the recurrence risk is higher and the possibility of cure is reduced as locoregional failures are incurable.

Second, are we really sure that there is a role for MIS in RPS? These tumors are almost invariably large at presentation, with a median size of $20 \mathrm{~cm} .{ }^{5,6}$ Indeed MIS was used for tumors smaller, on average, than those treated with open surgery $(10.5$ vs. $17 \mathrm{~cm}$ ); however, in the MIS group, the upper interquartile range value was $18.5 \mathrm{~cm}$. What is the real reason for approaching an $18.5 \mathrm{~cm}$ tumor with MIS, when the incision to remove the specimen will inevitably be much longer (at least as long as the longer of the two cross-sectional dimensions, plus any associated peripheral organs)? The authors demonstrated a 1-day gain 
in the length of hospital stay, but since recurrence data are not available in NCDB, it is unclear if oncologic outcomes are equivalent. In general, the percentage of tumors below $10 \mathrm{~cm}$ is low regardless of the histological subtype. Furthermore, to those not familiar with tumor biology of the most common RPS subtype, i.e. liposarcoma, misreading a computed tomography (CT) scan may lead the surgeon to think a MIS approach is feasible when it is not. We commonly see misinterpretation of patients presenting with well-differentiated and dedifferentiated liposarcoma, in whom the dedifferentiated (solid) component is resected, while the well-differentiated (lipomatous) component is left behind, often due to the lack of recognition. A supposedly successful MIS removing the dedifferentiated component would instead inevitably leave behind all the well-differentiated disease, resulting in an incomplete (R2) resection (Fig. 1). This again underlines the importance of specific expertise in the disease to make the right decision and give the patient a higher chance of cure.

We do not wish to completely dismiss the use of MIS for RPS. Similar skepticism met the introduction of MIS for cholecystectomy and colon cancer resections. For the latter, a carefully designed phase III trial measuring not only the length of stay (shorter) but, more importantly, oncologic parameters such as extent of lymphadenectomy, bowel margins, and recurrence and survival rates (all similar) ultimately led to the acceptance of laparoscopic colectomy for colon cancer ${ }^{9}$ (COST trial). The authors emphasize the need for a higher level of evidence in the discussion, stressing that any attempt to adopt MIS for RPS must first be evaluated in clinical trials to demonstrate equivalent outcomes. We agree that, occasionally, MIS can be considered. Frankly though, designing such a trial would be challenging. A trial would have to have strict eligibility criteria, including size (e.g. maximal size $10 \mathrm{~cm}$ ) and reasonable histological subtype. Among the different variants of RPS, solitary fibrous tumor is potentially the one most likely candidate for MIS as it is, on average, smaller, with a more limited recurrent risk and a lesser need for an extended resection. Other RPS subtypes would rarely be candidates for MIS. The $12 \%$ rate of MIS, and its growing trend over time recorded in the NCDB without any proof of oncologic appropriateness, is worrisome and underlines how often these patients may be inadequately treated.

Third, it is very important to remember that the best chance of resection with curative intent is at the time of primary presentation. ${ }^{8}$ The individual management plan should be determined following discussion at a multidisciplinary sarcoma case conference, with presentation of both imaging and pathologic findings. As described above, biologic behavior, response to treatment, and clinical outcomes vary by histological subtype of RPS. The management plan, including a plan for, and approach to, resection and use of neoadjuvant treatment, should be developed in recognition of this. Macroscopically complete resection (R0/R1) is the cornerstone of management. Surgery should be aimed at achieving an R0/R1 resection with a single specimen encompassing the tumor and involved contiguous organs and minimizing microscopically positive margins. This is best achieved by variably resecting the tumor en bloc with a subset of adherent but not infiltrated structures, including peritoneum, psoas, etc. Preservation of specific organs (e.g. kidney, duodenum, pancreas, etc.) should be considered on an individualized basis, and mandates a specific expertise in the biology of the disease to make the appropriate decisions. Judgment must be used in deciding which neurovascular structures to

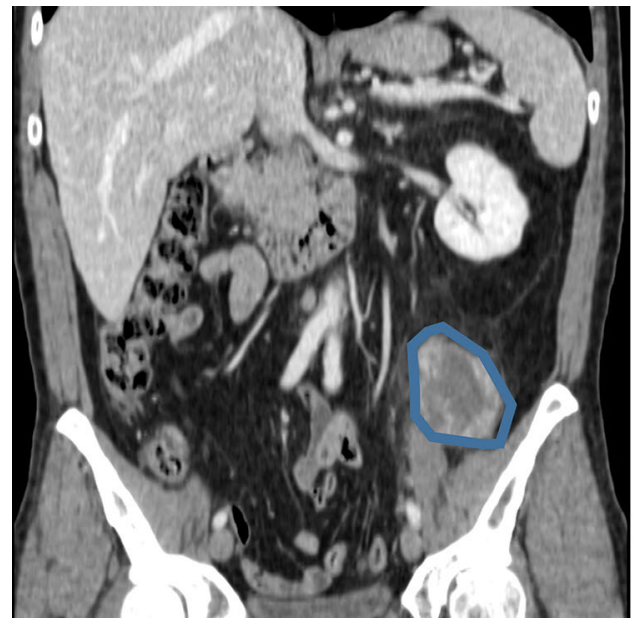

FIG. 1 Contrast-enhanced CT scan, venous phase, coronal view, before and after incomplete laparoscopic resection: the dedifferentiated component (blue line, left panel) was successfully

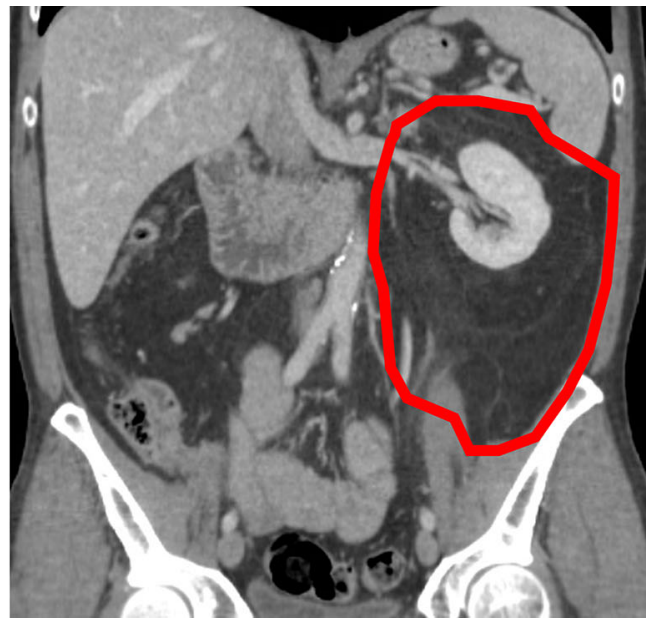

removed laparoscopically, leaving behind all the well-differentiated component (red line, right panel) still visible on the postoperative CT scan. $C T$ computed tomography 
sacrifice, weighing the potential for local and distal control in the context of tumor histology against the potential for long-term dysfunction. Performing such multivisceral resections, and the multilayered decision-making required, cannot be done routinely via MIS. Resection of RPS requires technical expertise in multiple sites throughout the abdominal and pelvic cavity, including the handling of large vessels. Single organ/site expertise, as well as MIS expertise, are not sufficient. The open surgical technique is fairly complex ${ }^{10}$ and an equivalent MIS approach does not exist, and will likely never exist. The use of MIS should be an exception rather than an option.

Given their rarity, what evidence do we need to draw firm conclusions about the best approach to RPS? The incidence of RPS is $<0.5 / 100,000 /$ year. As mentioned above, a clinical trial similar to the COST trial ${ }^{9}$ is unlikely to accrue, both due to the rarity of overall presentation and the location and heterogeneity of subtypes. We may discuss how 'standard treatment' can be defined in rare tumors. Clearly, we must take advantage of any evidence we have by fairly balancing it against consensus within the medical community. National, international, and even global collaborations should be pursued to make more studies possible. Prospectively maintained databases, institutional/multi-institutional registries, and biobanks for medical research are some of the cornerstones for making progress in rare cancers.

In summary, MIS for RPS cannot and should not be routinely employed. This article demonstrates a short hospital length of stay with MIS, but does not prove that MIS is equivalent to open surgery in terms of oncologic outcome, as was demonstrated for colon cancer. There is no place for MIS in the management of RPS, unless decided otherwise for specific presentations after multidisciplinary discussion. This should be the take-home message from the manuscript by Gani and colleagues. ${ }^{1}$ Just because MIS can be performed for some RPSs does not mean that it should be. It is not standard of care.

\section{REFERENCES}

1. Gani F, Goel U, Blair AB, et al. Minimally invasive versus open primary resection for retroperitoneal soft tissue sarcoma: a propensity-matched study from the national cancer database. Ann Surg Oncol. Epub 31 May 2018.

2. Derbel O, Heudel PE, Cropet C, et al. Survival impact of centralization and clinical guidelines for soft tissue sarcoma (A prospective and exhaustive population-based cohort). PLoS One. 2017;12(2):e0158406.

3. Blay JY, Soibinet P, Penel N, et al. Improved survival using specialized multidisciplinary board in sarcoma patients. Ann Oncol. 2017;28(11):2852-2859.

4. Toulmonde M, Bonvalot S, Méeus P, et al. Retroperitoneal sarcomas: patterns of care at diagnosis, prognostic factors and focus on main histological subtypes: a multicenter analysis of the French Sarcoma Group. Ann Oncol. 2014;25(3):735-742.

5. Gronchi A, Strauss DC, Miceli R, et al. Variability in patterns of recurrence after resection of primary retroperitoneal sarcoma (RPS): a report on 1007 patients from the multi-institutional collaborative RPS Working Group. Ann Surg. 2016;263(5):1002-1009.

6. Tan MC, Brennan MF, Kuk D, et al. Histology-based classification predicts pattern of recurrence and improves risk stratification in primary retroperitoneal sarcoma. Ann Surg. 2016;263(3):593-600.

7. Bonvalot S, Gaignard E, Stoeckle E, et al. Survival impact of surgical management in reference centers for retroperitoneal sarcoma: a nationwide study of FSG-GETO and NETSARC [abstract no. 11568]. J Clin Oncol. 2018;36(Suppl).

8. Management of primary retroperitoneal sarcoma (RPS) in the adult: a consensus approach from the Trans-Atlantic RPS Working Group. Ann Surg Oncol. 2015;22(1):256-263.

9. A comparison of laparoscopically assisted and open colectomy for colon cancer. The Clinical Outcomes of Surgical Therapy Study Group. N Engl J Med. 2004;350:2050-2059.

10. Bonvalot $\mathrm{S}$, Raut CP, Pollock RE, et al. Technical considerations in surgery for retroperitoneal sarcomas: position paper from E-Surge, a master class in sarcoma surgery, and EORTC-STBSG. Ann Surg Oncol. 2012;19(9):2981-2991. 